\title{
An Assessment of Clinical and Functional Outcomes in the Patients Undergoing Total Knee Arthroplasty during Postoperative Period
}

\author{
Aakaash Sethuraman Venkatesan ${ }^{1}$, Perur Jayasankar $^{2}$, Sudhakar Williams $^{3}$
}

\begin{abstract}
Background and objective: Patient-reported outcome measures continue to play an important role in assessing the performance and determining the comparative effectiveness of total knee arthroplasty. Patient's satisfaction can be influenced by many factors, such as, residual pain, postoperative functionality, and the presence of postoperative complications and hence we evaluated clinical and functional outcomes following total knee arthroplasty. This study was conducted to bust the myth of postoperative pain and disability following a total knee replacement.

Materials and methods: A prospective observational cohort study was conducted among the patients who underwent primary total knee arthroplasty in the Department of Orthopaedics in the Sundaram Medical Foundation during the study period of March 2017 and January 2018. A total of 30 cases were included. During their follow-up, patient's outcomes were assessed using Western Ontario and McMaster Universities Osteoarthritis Index (WOMAC) and American Knee Society Scores (AKSS). Data were analyzed using SPSS v.17.

Results: At the end of 6 months, $76.7 \%$ of participants were satisfied with their outcome. Significant improvement was noted in both clinical ( $p$ $=0.000)$ and functional outcome $(p=0.000)$ of AKSS and total WOMAC scores $(p=0.007)$ during the follow-up at the sixth month. Also, there was a significant difference in AKSS scores noted with respect to age but other parameters like duration of illness and type of arthritis were not significant.

Conclusion: The majority of study subjects were satisfied with the clinical and functional outcome based on WOMAC and AKSS, which in turn encouraged them to undergo total knee replacement of the other knee.

Clinical significance: From our study, we could determine the importance of patient- and clinician-reported outcome measures in predicting the satisfaction of the patient following total knee replacement. Assessment of a patient planned for total knee replacement, with the outcome scores both preoperatively and postoperatively will give us a brief idea on how better the patient will fair following surgery and will also help us in the rehabilitation of the patient accordingly.

Keywords: American Knee Society Score, Clinical outcome, Functional outcome, Total knee arthroplasty, Western Ontario and McMaster Universities Osteoarthritis Index.

Journal of Orthopedics and Joint Surgery (2020): 10.5005/jp-journals-10079-1027
\end{abstract}

\section{INTRODUCTION}

Total knee replacement is a common orthopedic surgical procedure, performed to treat the cases of severe arthritis of the knee joint either due to osteoarthritis (OA) or inflammatory arthropathy. Osteoarthritis is conceived as a process that is initiated and perpetuated by mechanical stress. ${ }^{1}$ As like all diseases, OA is a consequence of the interplay between host and environmental factors. It can be conceived as resulting from excessive joint loading resulting from perturbations of joint biomechanics that initiates a microinflammatory process, which in turn results in loss of joint homeostasis. ${ }^{1}$

When there is cartilage damage in either medial, lateral compartment, or patellofemoral joint, severe pain with deformity develops subsequently necessitating a total joint replacement. When anti-inflammatory medications, lifestyle modification, and physiotherapy do not succeed in relieving symptoms of pain and improving functional capacity, joint replacement is considered as the last resort.

The surgical management of OA varies from soft tissue interposition arthroplasty to surface replacement arthroplasty, especially total knee replacement. The primary aim of total knee replacement is to relieve pain, to reduce stiffness, and to increase the range of movements. A successful outcome needs precise
${ }^{1}$ Department of Orthopaedics, University Hospital, Llandough, Cardiff and Vale Health Board, Cardiff, United Kingdom

${ }^{2,3}$ Department of Orthopaedics, Sundaram Medical Foundation, Chennai, Tamil Nadu, India

Corresponding Author: Aakaash Sethuraman Venkatesan, Department of Orthopaedics, University Hospital, Llandough, Cardiff and Vale Health Board, Cardiff, United Kingdom, Phone: +447448540756, e-mail: aakaashsv86@gmail.com

How to cite this article: Venkatesan AS, Jayasankar P, Williams S. An Assessment of Clinical and Functional Outcomes in the Patients Undergoing Total Knee Arthroplasty during Postoperative Period. J Orth Joint Surg 2020;2(2):57-61.

Source of support: Nil

Conflict of interest: None

surgical technique, sound implant design, and kinematics with appropriate materials and compliance of patients in rehabilitation. The use of accurate instrumentation and an understanding of the basic principles inherent to the instruments are necessary to implant reproducibly well-aligned prostheses. Computer-assisted navigation is being used by some surgeons to try to improve the reproducibility of component alignment. ${ }^{2}$ 
The value of total knee replacement is being subjected to increased scrutiny, due to the cost of the procedure to the healthcare system and numerous reports suggesting disturbing rates of patient dissatisfaction with the outcome of this procedure. ${ }^{3}$ Moreover, given the ubiquity of OA of the knee and the demand for prosthetic replacement, increasing pressure is being placed on healthcare providers to demonstrate the benefits and costeffectiveness of total knee replacement in an objective manner. ${ }^{4}$ This has prompted a recent push to quantify the success of these procedures using patient-reported outcome measures (PROMs).

The outcomes of total knee arthroplasty can be assessed with various methods; implant survivorship, image-based assessment, clinical assessment, and PROMs. While the first three modalities are objective in nature, the patient-reported outcome can provide a subjective measure of the patient's perception of the success of an intervention. ${ }^{5}$

Patient-reported outcome measures continue to play an important role in assessing the performance and determining the comparative effectiveness of total knee arthroplasty, their use is seeing a transition from simply a research setting to that of clinical practice. It is expected their use will play a major role in benchmarking outcomes in total knee arthroplasty. ${ }^{6}$ The patient satisfaction is influenced by many factors, such as, residual pain, postoperative functionality, and the presence of postoperative complications and hence it differs with place and time.

In our study, we evaluated clinical and functional outcomes following total knee arthroplasty, which include both clinicianand patient-related outcome measures, to assess the correlation between patient satisfaction and traditional physician-based scoring systems.

\section{Materials and Methods}

\section{Patients}

A prospective observational cohort study was conducted among the patients who underwent total knee arthroplasty for both inflammatory arthritis and OA in the Department of Orthopaedics in the Sundaram Medical Foundation during the study period of March 2017 and January 2018 were included in the study. Patients who underwent revision arthroplasty, ipsilateral lower limb trauma, ipsilateral hip arthroplasty, and cases with preexisting neurological or muscular disorders were excluded from the study. A total of 30 cases were included in the study after assessing the inclusion and exclusion criteria.

\section{Informed Consent}

The individual participants were explained about the study and they were also assured that their identity would be kept strictly confidential and they have the option to refuse participation in the study. Written informed consent was obtained from the study participant's parents before the interview. Every effort was made, to be sure that all information collected from the participants remain confidential.

All the patients underwent total knee arthroplasty and the intra surgical and immediate postoperative was uneventful. Patients were followed up in the third-and sixth-month postoperative period, respectively, in the orthopedic outpatient department. During their follow-up, patients were assessed clinically of their range of movements and mobility status, and the current functional scoring was done using both Western Ontario and McMaster
Universities Osteoarthritis Index (WOMAC) and American knee society scores (AKSS), respectively. Plain radiographs of both knee antero posterior (AP) and lateral were taken at the follow-up visit and radiological assessment was done.

\section{Assessment Scales}

Western Ontario and McMaster Universities Osteoarthritis Index: WOMAC OA index is a widely used self-report outcome measure in patients with lower limb OA that was introduced by Bellamy and Buchanan. The original score with 5-point Likert response categories consists of 24 questions covering three dimensions: Pain (5 questions), stiffness (2 questions), and function (17 questions). Each one of the questions is graded on a scale from 0 to 4 points. The WOMAC has been extensively tested for validity, reliability, feasibility, and responsiveness for measuring changes after different OA interventions and has also been evaluated in an electronic form. Western Ontario and McMaster Universities Osteoarthritis Index scores were linearly transformed to a 0-100 scale with higher scores indicating more severe impairment.

American Knee Society Score was published by the American Knee Society in 1989 which is divided into two components. The first assesses the knee clinically through the physical examination (Clinical AKSS- "Knee Score"), and the second assesses the individual's functionality (Functional AKSS- "Function Score"), while both attain a total of 100 points each. The objective of this separation was to make the scoring of the Clinical AKSS Independent of the Functional AKSS, not being influenced by any other variables.

\section{Data Analysis}

Data entry was done using Microsoft excel and data were analyzed using Statistical Package for Social Sciences (SPSS) version 17. All descriptive data were described as frequency, percentage, mean, and standard deviation. The significant differences in the mean scores were tested using paired $t$-test and the independent sample $t$-test, appropriately. Statistical testing was undertaken considering $p$ values $<0.05$ to be significant.

\section{Results}

The mean age of the study participants was $68 \pm 7.2$ years. Also, $63 \%$ of the study population aged $<70$ years and $37 \%$ of them aged $>70$ years. Only three patients (10\%) were males and the majority (90\%) were females. Right side total knee arthroplasty (TKR) was more common (60\%) in this study. More than half of the participants (53\%) underwent total knee replacement within 5 years of onset of pain and the overall mean duration pain was found to be $4.1 \pm$ 2.25 years. All the patients recruited were in stage IV of the disease. On radiological examination (X-ray), none of the patients had evidence of lysis. Majority of the patients (77\%) presented with a varus deformity followed by valgus deformity (13\%) and fixed flexion deformity (10\%). Among the 30 cases included, $83.3 \%$ had $\mathrm{OA}$ and $16.7 \%$ had rheumatoid arthritis. Postoperative X-ray knee both AP and lateral view of a female patient is shown in Figure 1.

\section{Knee Society Clinical Scores}

At the end of 6 months, based on knee society clinical scores, $76.7 \%$ of the population were satisfied with their outcome $(30 \%$ had excellent scores and $46.7 \%$ had good scores after 6 months) and were willing to get their other knee operated on as well. $3.3 \%$ of the study population had a poorer outcome measure. The mean AKSS 
score at third and sixth months showed a statistically significant improvement in all components as shown in Table 1.

\section{WOMAC Scores}

Western Ontario and McMaster Universities Osteoarthritis Index total scores are a summation of various modalities assessed like symptoms, stiffness, pain, and function scores, this bar diagram depicts the improvement in total scores at the end of 6 months. $76.7 \%$ of the study population had a total WOMAC score $<30$ indicating good patient satisfaction after total knee replacement. The less the WOMAC scores better the outcome. The mean WOMAC score at third and sixth months showed a statistically significant improvement in the symptoms and the total WOMAC scores, whereas there was no significant difference in the mean scores with respect to stiffness, pain, and functions as shown in Table 2. Also, the clinical flexion and extension of the knee by a female patient are shown in Figure 2.

Factors, such as, age, duration of pain in years, type of arthritis, the gender of the patient, and their significance in assessing the outcomes following total knee replacement in the forms of WOMAC and Knee society scores, were analyzed. Among which clinical and function AKSS scores showed a statistically significant difference
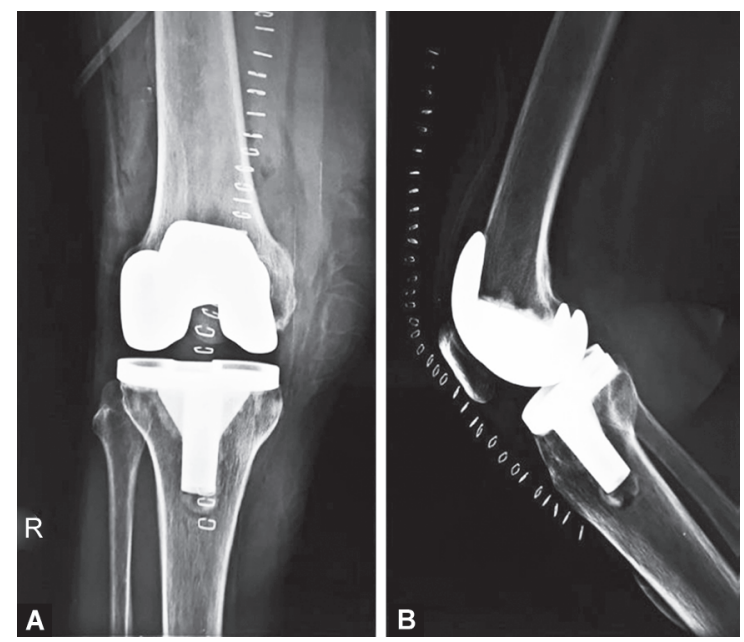

Figs $1 \mathrm{~A}$ and $\mathrm{B}$ : Postoperative X-ray of a female patient: (A) Anteroposterior view; (B) Lateral view with respect to age. All other scores were found to be insignificant with respect to the duration of pain and type of arthritis as shown in Table 3.

\section{Discussion}

Osteoarthritis of the knee is a relatively prevalent condition that can progress to a point where it is debilitating. It adversely affects the patient's quality of life due to pain and decreased function. While there are many modes of treatment, including conservative and surgical management, the definitive method of treatment is joint replacement. In this regard, TKA is safe and effective in addressing end-stage $O A$ of the knee. This surgical procedure has become more common due to its effectiveness in alleviating pain and improving function and will continue to play a key role in the management of OA. ${ }^{7}$ Many outcome measures have been used to measure the efficacy of TKA, including subjective and objective scales. Although

Table 1: Mean difference in American Knee Society Scores (AKSS) at the third and sixth months

\begin{tabular}{lccl}
\hline Components of AKSS & At 3rd month & At 6th month & p value \\
\hline Pain & $39.33 \pm 5.68$ & $42.50 \pm 3.65$ & $0.000^{*}$ \\
Flexion & $20.33 \pm 1.44$ & $21.07 \pm 1.53$ & $0.001^{*}$ \\
Total clinical score & $71.7 \pm 9.68$ & $75.37 \pm 7.13$ & $0.000^{*}$ \\
Walking & $27.67 \pm 10.72$ & $30.67 \pm 9.44$ & $0.017^{*}$ \\
Climbing stairs & $30.83 \pm 4.56$ & $34.83 \pm 6.22$ & $0.000^{*}$ \\
Total functional score & $52.50 \pm 15.07$ & $63.00 \pm 14.36$ & $0.000^{*}$ \\
\hline
\end{tabular}

*Significant

Table 2: Mean difference in Western Ontario and McMaster Universities Osteoarthritis Index (WOMAC) scores at the third and sixth months

\begin{tabular}{lrrl}
\hline $\begin{array}{l}\text { Components of } \\
\text { WOMAC }\end{array}$ & At 3rd month & At 6th month & p value \\
\hline Symptoms score & $9.37 \pm 2.31$ & $7.07 \pm 2.85$ & $0.001^{*}$ \\
Stiffness score & $1.45 \pm 0.82$ & $1.76 \pm 1.02$ & 0.095 \\
Pain score & $6.73 \pm 3.40$ & $6.77 \pm 2.64$ & 0.957 \\
Function score & $9.87 \pm 3.85$ & $9.93 \pm 3.29$ & 0.901 \\
Total WOMAC score & $27.40 \pm 6.43$ & $25.03 \pm 6.23$ & $0.007^{*}$ \\
\hline
\end{tabular}

*Significant
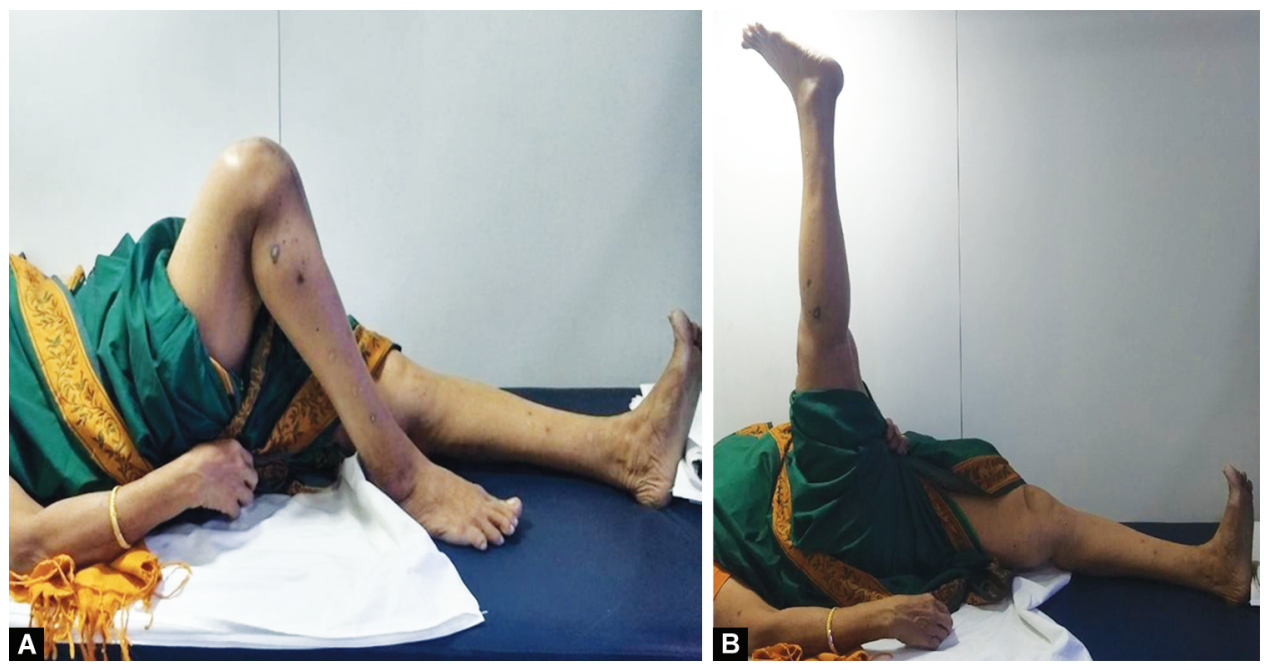

Figs $2 \mathrm{~A}$ and $\mathrm{B}$ : Postoperative flexion and extension of a female patient at the sixth month 
Table 3: Difference in mean scores at the sixth month with respect to age, duration of pain, and type of arthritis

\begin{tabular}{llll}
\hline Categories & WOMAC & Clinical AKSS & Functional AKSS \\
\hline Age group & & & \\
$\leq 70$ years $(N=19)$ & $23.58 \pm 6.51$ & $79.21 \pm 4.44$ & $68.95 \pm 7.74$ \\
$>70$ years $(N=11)$ & $27.55 \pm 5.02$ & $68.73 \pm 5.96$ & $52.73 \pm 17.51$ \\
$p$ value & 0.093 & $0.000^{*}$ & $0.002^{*}$ \\
Duration of pain & & & \\
$\leq 5$ years $(N=16)$ & $24.50 \pm 5.71$ & $76.69 \pm 7.39$ & $64.69 \pm 14.43$ \\
$>5$ years $(N=14)$ & $25.64 \pm 6.94$ & $73.86 \pm 6.78$ & $61.07 \pm 14.56$ \\
$p$ value & 0.625 & 0.286 & 0.501 \\
Type of arthritis & & & \\
Rheumatoid arthritis $(N=25)$ & $25.12 \pm 6.82$ & $75 \pm 7.52$ & $62 \pm 15.13$ \\
Osteoarthritis $(N=5)$ & $24.6 \pm 1.51$ & $77.2 \pm 4.91$ & $68 \pm 9.08$ \\
$p$ value & 0.868 & 0.539 & 0.403 \\
\hline
\end{tabular}

*Significant. AKSS, American Knee Society Scores; WOMAC, Western Ontario and McMaster Universities Osteoarthritis Index

the use of these scales may indicate improvement in the majority of patients, there remains a significant proportion of patients who are dissatisfied with the outcome of the TKA. The dissatisfaction rate for TKA has been reported to be as high as 19\%. ${ }^{7}$ Additionally, it has been suggested that clinician-assessed outcomes may not always match those reported by patients. In other words, there is a need for patient-reported outcomes and satisfaction levels to be included in the routine postoperative assessment of patients who undergo TKAs.

Among the study participants, $83 \%$ of patients had OA and $17 \%$ of patients had rheumatoid arthritis. There was no significant difference in the outcome of our study based on the type of arthritis. A study done by Alexander et al. ${ }^{8}$ on the functional outcome of total knee replacement in young patients based on knee society scores had 24 patients with a 10 year follow-up of which 17 patients had $\mathrm{OA}$ and 7 patients had rheumatoid arthritis. They also concluded that there was no statistical difference in the functional outcome after 10 years in relation to the type of arthritis.

The mean age group of our study population was 68 years in an age group ranged from 58 to 80 years. Giesinger et al. ${ }^{5}$ conducted a study on comparison of outcome measures following total knee arthroplasty on 98 knees, had a mean age group of 68 years.

In our study, there were more number of women than men who underwent total knee replacement in our study, of which two women went onto have a bilateral replacement. There was no significant difference in the outcome measures and the gender of the study population. In a study done by Robert ${ }^{9}$ on various measuring tools for functional outcomes in total knee arthroplasty, 843 TKA's for a mean follow-up of 9.5 years, found no statistical difference in pre- and postoperative scores with respect to gender.

Baker et al. ${ }^{10}$ conducted a study on the role of pain and function in determining patient satisfaction after total knee replacement based on the Oxford knee scores concluded saying that female gender and a primary diagnosis of $\mathrm{OA}$ were found to be predictors of low levels of patient satisfaction.

In our study, we have categorized our patients into those who underwent total knee replacement within 5 years of pain symptoms and those who underwent after 5 years of pain symptoms. We found from our study that there was no statistical significance on the outcome scores both in Knee society scores and WOMAC scores at the end of 6 months of total knee replacement. In the literature review done during the study period, none of the literature showed any significance on the outcome scores based on the duration of the pain sustained.

\section{Knee Society Scores}

Among the study participants, $76.7 \%$ of cases were satisfied with the outcome at the end of 6 months, out of which $30 \%$ had excellent scores and $56.7 \%$ had good outcome scores. There was a significant change over time in pain, flexion range, and the final score at the end of 6 months $(p=0.000)$. Patient-reported knee functional score at the end of 6 months had $66.7 \%$ of the population satisfied with the outcome. Similarly, there was a significant improvement in walking $(p=0.0017)$, stair climbing $(p=0.000)$ at the end of 6 months.

The findings of our study are consistent with the study conducted by Giesenger et al. ${ }^{5}$ with 98 cases who underwent TKAs reported similar mean age and female predominance and they reported $49.5 \%$ improvement in knee society scores during the follow-up period.

A study done on functional outcome based on knee society scores in young patients with a mean follow-up of 10 years by Alexander et al. ${ }^{8}$ showed a statistically significant difference in pain $(p=0.025)$, American knee society score $(p<0.01)$, and function ( $p<0.001)$, according to their study functional scores are better for younger knees. A study conducted by Johannes et al. ${ }^{11}$ on the threshold for treatment success reported $76.8 \%$ of patient satisfaction at the end of 12 months and Thambiah et al. ${ }^{7}$ reported $92.8 \%$ patient satisfaction after total knee replacement.

\section{WOMAC Scores}

In our study, the WOMAC assessment was done at 3 and 6 months, respectively. The patient's symptoms, pain, function, and stiffness were taken into consideration, as a part of the scoring system. $76.7 \%$ of the population had a satisfactory total score at the end of 6 months. There was a significant improvement in symptoms ( $p=$ $0.001)$ and the total score $(p=0.007)$ at the end of 6 months of total knee replacement. In our study, there was no significant difference in the WOMAC outcome score in relation to various factors, such as, the age of the patient, gender, type of arthritis, and duration of pain.

A study by Escobar et al. ${ }^{12}$ on 365 knees post-replacement with $75 \%$ female population with similar mean age showed an 
improvement in WOMAC pain scores from 55.56 to 31.62 after 6 months of surgery and functional limitations from 58.24 to 27.22.

A study by Bachmeier et al. ${ }^{13}$ on comparison of outcomes in OA patients undergoing total knee replacement reported a $53 \%$ reduction in pain, a $43 \%$ reduction in stiffness, and improvement in function at the end of 1 year.

A study by Anderson et al. ${ }^{14}$ examined the patient's satisfaction and reported a significant association between decreased function and increased pain scores during the postoperative period using the WOMAC scores.

\section{Conclusion}

From this study, we conclude that patient satisfaction is correlated with postoperative Knee society score and WOMAC score, majority of study subjects were satisfied with the outcome, which in turn encouraged them to undergo total knee replacement of the other knee and we were able to encourage other patients with severe OA to undergo the procedure to have a pain-free mobilization.

\section{Ethical Approval}

This study was registered with the Institutional Human Ethical Committee.

\section{References}

1. Collins NJ, Prinsen CAC, Christensen R, et al. Knee injury and osteoarthritis outcome score: systematic review and meta analysis of measurement properties. Osteoarthritis Cartilage 2016;24(8):13171329. DOI: 10.1016/j.joca.2016.03.010.

2. Kim RH, Scott WN. Operative techniques: total knee replacement. Philadelphia: Saunders-Elsevier; 2009. pp. 91-103.
3. Noble PC, Conditt MA, Cook KF, et al. The john insall award:patient expectations affect satisfaction with total knee arthroplasty. J Clin Orthop Relat Res 2006;452:35-43. DOI: 10.1097/01. blo.0000238825.63648.1e.

4. Jones CA, Beaupre LA, Johnston DW, et al. Total joint arthroplasties:current concepts of patient outcomes after surgery. J Rheum Dis North Am 2007;33(1):71-86. DOI: 10.1016/j.rdc.2006.12.008.

5. Giesinger K, Hamilton DF, Jost $B$, et al. Comparative responsiveness of outcome measures for total knee arthroplasty. Osteoarthritis Cartilage 2014;22(2):184-189. DOI: 10.1016/j.joca.2013.11.001.

6. Hossain FS, Konan S, Patel S, et al. The assessment of outcome after total knee arthroplasty: are we there yet? Bone Joint J 2015;97(1):3-9.

7. Thambiah MD, Nathan S, Seow BZX, et al. Patient satisfaction after total knee arthroplasty: an asian perspective. Singapore Med J 2015;56(5):259-263. DOI: 10.11622/smedj.2015074.

8. Wood AM, Keenan ACM, Arthur CHC, et al. The functional outcome of total knee replacement in young patients: $\mathrm{A} 10$ year matched case control study. Open J Orthop 2013;3(1):128-132.

9. Bourne RB. Measuring tools for functional outcomes in total knee arthroplasty. Clin Orthop Relat Res 2008;466(6):2634-2638.

10. Baker PN, Van der Meulen JH, Lewsey J, et al. The role of pain and function in determining patient satisfaction after total knee replacement. J Bone Joint Surg Br 2007;89(7):893-900.

11. Giesinger JM, Hamilton DF, Jost B, et al. WOMAC,EQ-5 Dand knee societys core thresholds for treatment success after total knee arthroplasty. J Arthroplasty 2015;30(12):2154-2158.

12. Escobar A, Quintana JM, Bilbao A, et al. Responsiveness and clinically important differences for the WOMAC and SF-36 after total knee replacement. Osteoarthritis Cartilage 2006;15(3):273-280.

13. Bachmeier CJM, March LM, Cross MJ, et al. A comparison OF outcomes in osteoarthritis patients Undergoing total hip and knee replacement surgery. Osteoarthritis Cartilage 2001;9(2):137-146.

14. Anderson JG, Wixson RL, Tsai D, et al. Functional outcome and patient satisfaction in total knee patients over the age of 75. J Arthroplasty 1996;11(7):831-840. 